\title{
Oestrogen promotes healing in a bacterial LPS model of delayed cutaneous wound repair
}

\author{
Rachel Crompton ${ }^{1}$, Helen Williams ${ }^{1}$, David Ansell ${ }^{1,2}$, Laura Campbell ${ }^{1}$, Kirsty Holden ${ }^{3}$, Sheena Cruickshank ${ }^{4}$ and \\ Matthew J Hardman ${ }^{1}$
}

Wound infection is a major clinical problem, yet understanding of bacterial host interactions in the skin remains limited. Microbe-derived molecules, known as pathogen-associated molecular patterns, are recognised in barrier tissues by pattern-recognition receptors. In particular, the pathogen-associated molecular pattern, lipopolysaccharide (LPS), a component of microbial cell walls and a specific ligand for Toll-like receptor 4, has been widely used to mimic systemic and local infection across a range of tissues. Here we administered LPS derived from Klebsiella pneumoniae, a species of bacteria that is emerging as a wound-associated pathogen, to full-thickness cutaneous wounds in C57/BL6 mice. Early in healing, LPS-treated wounds displayed increased local apoptosis and reduced proliferation. Subsequent healing progression was delayed with reduced re-epithelialisation, increased proliferation, a heightened inflammatory response and perturbed wound matrix deposition. Our group and others have previously demonstrated the beneficial effects of $17 \beta$-estradiol treatment across a range of preclinical wound models. Here we asked whether oestrogen would effectively promote healing in our LPS bacterial infection model. Intriguingly, co-treatment with $17 \beta$-estradiol was able to promote re-epithelialisation, dampen inflammation and induce collagen deposition in our LPS-delayed healing model. Collectively, these studies validate K. pneumoniae-derived LPS treatment as a simple yet effective model of bacterial wound infection, while providing the first indication that oestrogen could promote cutaneous healing in the presence of infection, further strengthening the case for its therapeutic use.

Laboratory Investigation (2016) 96, 439-449; doi:10.1038/labinvest.2015.160; published online 8 February 2016

Our skin is home to a diverse range of microorganisms, collectively termed the skin microbiota. ${ }^{1}$ Under homeostasis, these organisms cause little harm and can even be beneficial to health. However, our environment is filled with pathogenic organisms to which we have evolved a range of defence mechanisms. ${ }^{2,3}$ The skin provides a physical barrier producing antimicrobial peptides and even promoting the growth of commensal bacteria to prevent overpopulation of pathogens. Innate immune cells continually sample the local environment providing a robust immunological barrier. However, upon injury or disease the skin barrier can be breached, opportunistic bacteria become pathogenic and, in favourable conditions, grow and develop, leading to infection. ${ }^{4,5}$

Because of their aetiology, chronic wounds are particularly susceptible to infection. ${ }^{6}$ Staphylococcus aureus and Pseudomonas aeruginosa are the most studied wound pathogens; however, Klebsiella pneumoniae, a Gram-negative bacterium that colonises the skin, has been increasingly demonstrated as a wound pathogen present in sterile/surgical and infected wound sites, ${ }^{7}$ including combat wounds, ${ }^{8-11}$ burns $^{8,12}$ and chronic leg ulcers. ${ }^{13-16}$ Persistent wound infection is a major clinical problem and likely to be a key driver of local chronic inflammation. ${ }^{17-21}$ Indeed, clinical studies suggest up to $90 \%$ of diabetic foot ulcers that eventually require lower limb amputation are preceded by infection. $^{22,23}$

The host response to the presence of bacteria and other pathogens occurs via pattern-recognition receptors (PRRs) that detect specific microbial components and metabolites, known as pathogen-associated molecular patterns (PAMPs). PRRs can also recognise endogenous ligands produced by stressed, injured or damaged host cells, known as damage-associated molecular patterns (DAMPs). Activation of PRRs orchestrates the early immune response via induction of pro-inflammatory cytokines, antiviral pathways and

\footnotetext{
${ }^{1}$ The Healing Foundation Centre, Faculty of Life Sciences, The University of Manchester, Manchester, UK; ${ }^{2}$ The Centre for Dermatology Research, Institute of Inflammation and Repair, The University of Manchester, Manchester, UK; ${ }^{3}$ Epistem, Manchester, UK and ${ }^{4}$ Faculty of Life Sciences, The University of Manchester, Manchester, UK Correspondence: Dr MJ Hardman, PhD, The Healing Foundation Centre, Faculty of Life Sciences, The University of Manchester, Oxford Road, AV Hill building, Manchester M13 9PT, UK.

E-mail: Matthew.j.hardman@manchester.ac.uk

Received 7 May 2015; revised 4 December 2015; accepted 11 December 2015
} 
activation of microbial killing mechanisms. ${ }^{24}$ Over recent years, the importance of PRRs has been recognised in wound healing. A number of wound-related cell types express PRRs, suggesting a role throughout the healing process. ${ }^{25-27}$ Clinical studies have identified increased Toll-like receptor (TLR) activity in human chronic wounds. ${ }^{28}$ In comparison, mechanistic studies in mouse models have shown that deletion of PRRs, specifically TLR $3,{ }^{29}$ TLR,${ }^{30}$ nucleotide-binding oligomerisation domain $2(\mathrm{NOD} 2)^{31}$ and downstream signalling component myeloid differentiation factor (MyD88), ${ }^{32}$ leads to impaired cutaneous healing. Furthermore, TLR2 expression is perturbed in diabetic mouse wounds. ${ }^{33}$ By contrast, stimulation of PRRs TLR3 (ref. 34) and TLR9 (ref. 30) have been shown to accelerate healing. The PRR TLR4 recognises lipopolysaccharide (LPS), a cell wall component of all Gram-negative bacteria. TLR4 stimulation by bacterial LPS has been shown to impede wound healing in a range of models. ${ }^{35,36}$ Moreover, persistent TLR4 activity has been identified in non-healing venous leg ulcers, while chronic in vivo stimulation of TLR4 in the skin has been shown to induce inflammation and fibrosis., ${ }^{37,38}$ This recent literature is in line with historic studies where systemic LPS administration has been used as a model of sepsis. ${ }^{39}$ Moreover, TLR4 has recently been shown to be essential to cutaneous wound repair, ${ }^{40,41}$ with TLR4 mutations associated with increased risk of developing a diabetic foot ulcer. ${ }^{42}$

The inflammatory response to TLR4 signalling has been widely studied; however, knowledge of the role of TLR4 in the cutaneous environment, particularly in response to bacterial Klebsiella colonisation of cutaneous wounds, is limited. Here we report the effect of $K$. pneumoniae-derived LPS administration to wounded skin. We identify delays to multiple aspects of the healing process, suggesting LPS administration provides a simple, reproducible model of bacterially delayed healing in mice. As with other current delayed healing murine models ${ }^{43}$ we suggest that our LPS model provides a clinically relevant means to dissect the host response to microorganisms in wound infection, with relevance to human chronic wounds. To test the suitability of this model, we co-treated with $17 \beta$-estradiol, the major oestrogen naturally occurring in mammals. Previous studies have shown a physiologically relevant role for oestrogen deficiency in delayed wound healing ${ }^{44}$ and demonstrated beneficial effects of oestrogen supplementation in both mice and humans. ${ }^{45-47}$ To our knowledge, the effects of oestrogen on bacterial infection of cutaneous wounds has yet to be studied. We thus assessed the effect of oestrogen treatment in our simple bacterial TLR4-stimulated wound model, showing for the first time that LPS-induced delayed healing can be reversed by the anti-inflammatory, pro-healing effects of oestrogen.

\section{MATERIALS AND METHODS}

\section{Animals and Wounding}

Following local ethics committee approval, animal studies were conducted in accordance with UK Home Office regulations. Six-7-week-old female C57/BL6 mice (purchased from Harlan Laboratories, UK) were anaesthetised and injected subcutaneously (LPS study) with $10 \mu \mathrm{g} \quad K$. pneumoniae-derived LPS (Sigma Aldrich, UK: L4268) or intradermally (LPS+E2 study) with $1 \mu \mathrm{g}$ LPS 24 and $2 \mathrm{~h}$ prior to wounding (5-8 mice per group). Corresponding groups were injected with vehicle (phosphate-buffered saline). Mice were then anaesthetised and wounded, following our established protocol. ${ }^{48}$ Briefly, two full-thickness $6-\mathrm{mm}$ excisional wounds or $1-\mathrm{cm}$ incisions were made through the injection site and left to heal by secondary intention. $17 \beta$-Estradiol was administered at the time of wounding via subcutaneous implantation of a 0.05-mg, 21-day, slow-release pellet (Innovative Research of America, Sarasota, FL, USA). Incisional wounds were excised at 1,3 or 7 days after wounding and bisected, half processed for histological analysis and half snap frozen and stored at $-80^{\circ} \mathrm{C}$ for biochemical analysis.

\section{Planimetric Analysis}

Excisional wounds were photographed daily (Finepix S5700 camera, Fujifilm, Bedford, UK) and wound closure was quantified as a percentage of initial wound area (image pro plus software) to determine temporal healing profile.

\section{Histological Analysis and Immunohistochemistry}

Histological sections were prepared from tissue fixed in 10\% buffered formalin and embedded in paraffin. In all, $5-\mu \mathrm{m}$ sections were stained by H\&E, Masson's Trichrome, Picro-Sirius Red or subjected to immunohistochemistry. Primary antibodies were used at the following concentrations: antineutrophil rat polyclonal $(1 \mu \mathrm{g} / \mathrm{ml}$; Thermo Scientific, UK:MA1-40038); anti-Mac-3 rat polyclonal $(15 \mu \mathrm{g} / \mathrm{ml} ; \mathrm{BD}$ Pharmingen, UK:553322); anti-F4/80 rat monoclonal $(10 \mu \mathrm{g} / \mathrm{ml}$; Invitrogen, UK); keratin $6(2 \mu \mathrm{g} / \mathrm{ml})$ and keratin $14(1 \mu \mathrm{g} / \mathrm{ml})$ rabbit polyclonal (Covance, UK:PRB-169 P and PRB-155 P), Ki67 rat monoclonal $(2 \mu \mathrm{g} / \mathrm{ml}$; Dako, UK: M7249), IL6 goat polyclonal $(4 \mu \mathrm{g} / \mathrm{ml} ; \mathrm{R} \& \mathrm{D}$ Systems, Abingdon UK: AF-406-NA), and TNF $\alpha$ rabbit polyclonal $(10 \mu \mathrm{g} / \mathrm{ml}$; Abcam, Cambridge UK: ab6671). All antibodies required citrate antigen retrieval, with the exception of F4/80 which required proteinase $\mathrm{K}$. Primary antibodies were detected using a Vectastain ABC Kit (Vector Laboratories, UK:PK-6104, PK-6101 or PK-6105), NovaRed substrate (Vector Laboratories, UK:SK-4800) or for F4/80 DAB and counterstained with haematoxylin. TUNEL staining was performed using an In Situ Cell Death Detection Kit (Roche, UK: 11684795 910), according to the manufacturer's protocol. Bright field images were captured using a Nikon Eclipse E400 microscope and SPOT insight camera (Image solutions, Preston, UK) or a Scanscope (Aperio,) with areas of interest analysed using the Imagescope software (Aperio). Fluorescent images were collected on an Olympus BX51 upright microscope using a $\times 10 / 0.30$ Plan Fln objective and captured using a Coolsnap EZ camera (Photometrics) 
through MetaVue Software (Molecular Devices). Picro-Sirius red staining was visualised using plane-polarised light (Leica), casting larger collagen fibres shades of red and thinner fibres green. Masson's Trichrome and Picro-Sirius red staining were quantified using Image J (http://rsb.info.nih.gov/ij). Total cell numbers, wound width, area and percentage re-epithelialisation were quantified using the Image Pro Plus software (MediaCybernetics, MD, USA). Wound width was determined by measuring the distance between wound margins. Wound area was calculated from wound margins below the eschar, extending to the level of the panniculus carnosus. Re-epithelialisation was expressed as a percentage of full closure.

\section{Proliferation Assay In Vitro}

Normal human epidermal keratinocytes (NHEK) isolated from juvenile foreskin (Promocell, UK: C-12002) were cultured according to the supplier's protocol and used at P5. Primary human keratinocytes for oestrogen studies were isolated from adult female abdominal skin, cultured in serum-free, phenol red-free media (Epilife medium (Thermo Scientific, UK: MEPICFPRF500) plus supplement kit $(\mathrm{S} 001 \mathrm{~K})$ ) and used at P2. Keratinocytes were seeded in 96-well plates at a density of $1 \times 10^{4}$ cells/well. Cells were treated with 0.001-10 $\mu \mathrm{g} / \mathrm{ml}$ LPS (Sigma Aldrich, UK: L4268), $10^{-7}$ M $17 \beta$-estradiol (Sigma Aldrich, UK: L4268) or TLR4 antagonist LPS-RS used at 100 times in excess of LPS (InvivoGen, Toulouse, FR: tlrl-prslps) and incubated at $37^{\circ} \mathrm{C}$, $5 \% \mathrm{CO}_{2}$ for $24 \mathrm{~h}$. Proliferation was measured, using the CellTiter96 AQueous MTS-based Kit (Promega, UK:G3582), relative to a calibration curve determined from wells known to contain between 0 and $1 \times 10^{5}$ cells, as per the manufacturer's protocol.

\section{Statistical Analysis}

Results are presented as mean \pm s.e.m. Statistical analysis was performed using IBM SPSS statistics v20. Differences were determined using two-tailed independent sample $t$-test (Mann-Whitney $U$-tests for nonparametric data), ANOVA with Tukey $\mathrm{Q}$ post hoc testing for $>2$ groups and considered significant at three levels: $P$-values of $<0.05,<0.01$, and $<0.001$.

\section{RESULTS}

\section{K. pneumoniae-Derived LPS Significantly Delays Cutaneous Healing}

To assess the effect of TLR4 activation via K. pneumoniae on wound healing, C57 BL6 mice were subcutaneously injected with LPS derived from K. pneumoniae, 2 and $24 \mathrm{~h}$ prior to excisional wounding. Planimetric analysis (Figures 1a and b) reveals significantly delayed healing in LPS-injected mice compared with vehicle (Day $5 P=0.002$, Day $6 P=0.030$, Day $7 P=0.025$, Day $8 P=0.020$, Day $9 P=0.028$ ). Histological analysis of LPS-injected, incisional wounds was performed to assess this phenotype at select time points (Figure 1c). Results reveal a delay in wound healing with LPS application, corresponding to the planimetry data, with greater wound width at 7 days after wounding $(P=0.048$; Figure 1d). Morphometric quantification of wound area further highlighted alterations in the temporal kinetics of healing, depicted by significantly reduced and increased wound area at 1 and 7 days, respectively, in LPS wounds $v s$ vehicle (Figure 1e). Qualitatively, LPS-treated wounds appeared vastly different to vehicle wounds with decreased cellularity and a necrotic appearance, particularly at day 3 after wounding (Figure 1c). Collectively, these results demonstrate that LPS significantly delays the early wound healing response.

\section{LPS Induces Early Cell Death and Inhibits Wound Edge Proliferation}

Bacterial induction of host cell death is widely reported, ${ }^{49}$ with LPS specifically implicated in cytotoxic effects. ${ }^{50,51}$ To determine whether this was an important mechanism in the observed phenotype, we assessed cell death (TUNEL staining) in LPS-injected and vehicle wounds. Apoptotic cells were increased, both in the wound edge dermis and within the wound granulation tissue, of LPS wounds at both 1 and 3 days after wounding (Figures 2a and b). Conversely, the percentage of proliferating, Ki67-positive, basal keratinocytes directly at the wound edge (field $1 ; 0-500 \mu \mathrm{m}$ from the wound edge) was significantly decreased by LPS administration 1 day after wounding (Figures $2 \mathrm{c}$ and $\mathrm{d}$ ). Recapitulating these in vivo results, a dose-dependent decline in proliferation of keratinocytes in vitro was also observed in NHEK cells after 24-h LPS stimulation (Figure 2e) with no difference in cell viability (data not shown), indicating a cell intrinsic effect. We co-treated NHEKs with LPS and the TLR4 antagonist LPS-RS and show that LPS-induced decrease in proliferation can be reversed by LPS-RS co-treatment (Figure 2f), suggesting TLR4 activity is necessary for the proliferation inhibition.

\section{Delayed Re-Epithelialisation of LPS Wounds}

In the light of delayed wound edge proliferative response, we asked whether re-epithelialisation could also be altered. Quantification of the epidermal keratinocyte marker, keratin 14, revealed that LPS administration significantly delayed re-epithelialisation $v s$ vehicle, evident at 3 days after wounding (Figures $3 \mathrm{a}$ and $\mathrm{b}$ ). We note that this delay was transient, with full wound closure of both treatment groups by day 7. No direct LPS effect on human keratinocyte migration was observed in in vitro scratch wounds after 24-h stimulation (data not shown); however, a dose-dependent reduction of human dermal fibroblast migration was evident following LPS treatment (data not shown). A common phenotype of experimental delayed healing models is an extended keratinocyte response. Upon wounding, keratinocytes switch to an activated state to contribute to the migration and proliferation required for re-epithelialisation and express keratin 6. Indeed, LPS administration resulted in greater extension of keratin 6 from the wound edge compared with 
vehicle ( $P=0.000038$; Figures $3 \mathrm{c}$ and $\mathrm{d})$. Consistent with these results, both the LPS and vehicle groups demonstrated high wound edge basal keratinocyte proliferation, but in cells remote to the wound edge (field 2; 500-1000 $\mu \mathrm{m}$ from the wound edge) proliferation was significantly higher with LPS administration (Figures $3 e$ and $f ; P=0.036$ ).

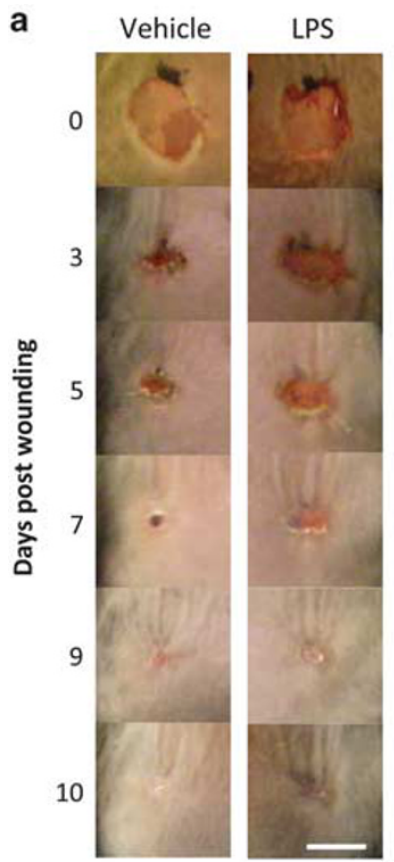

C

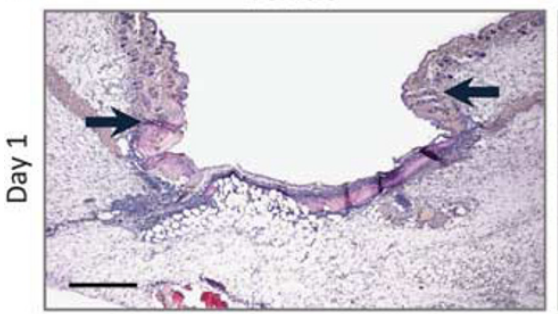

Vehicle
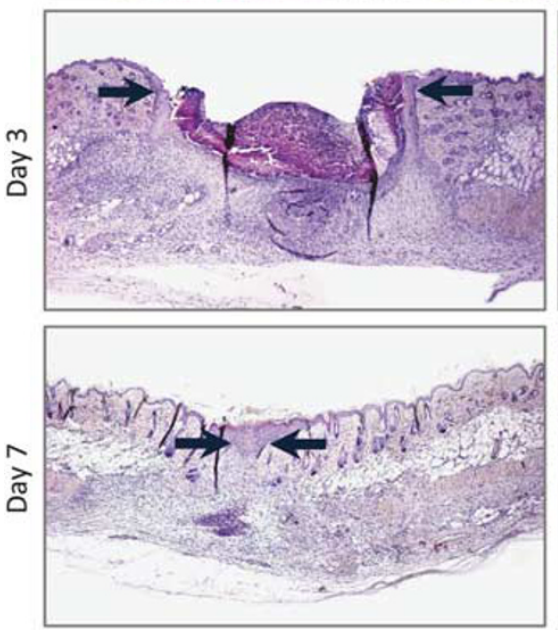

b

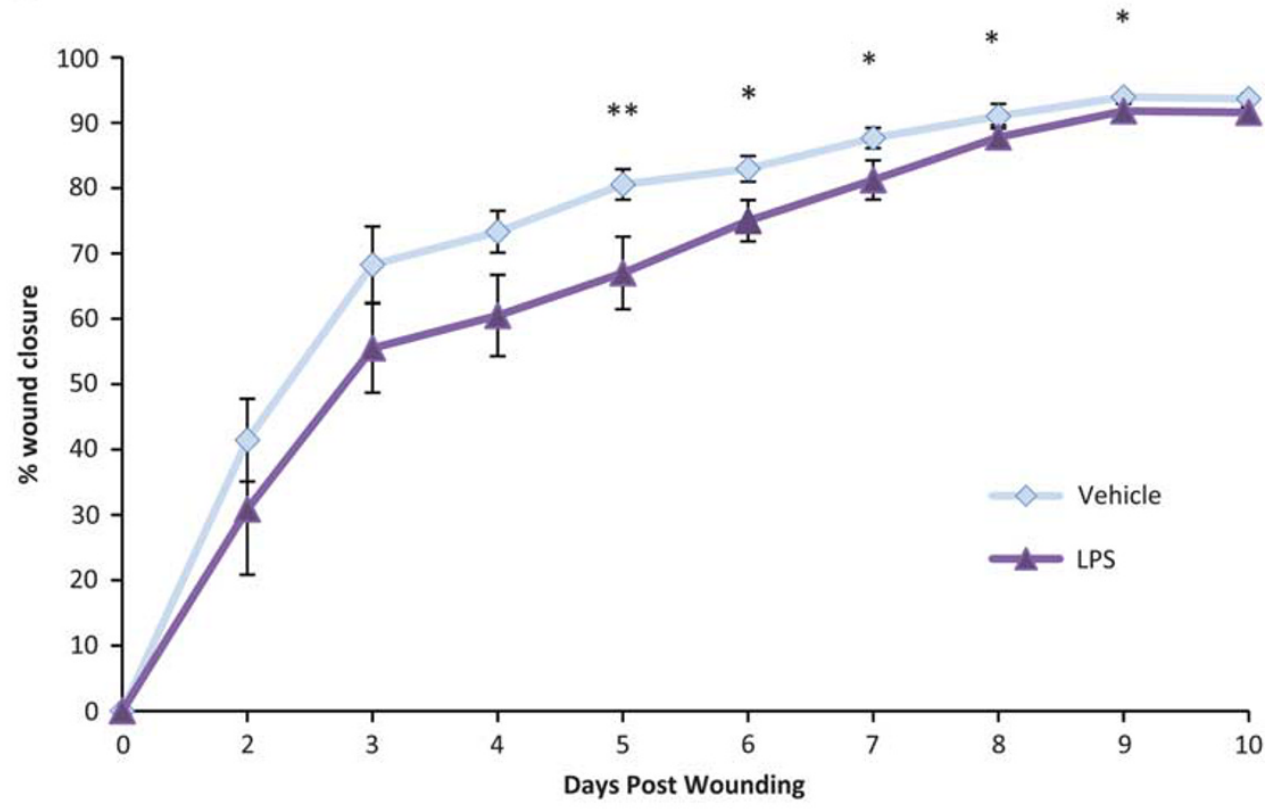

LPS
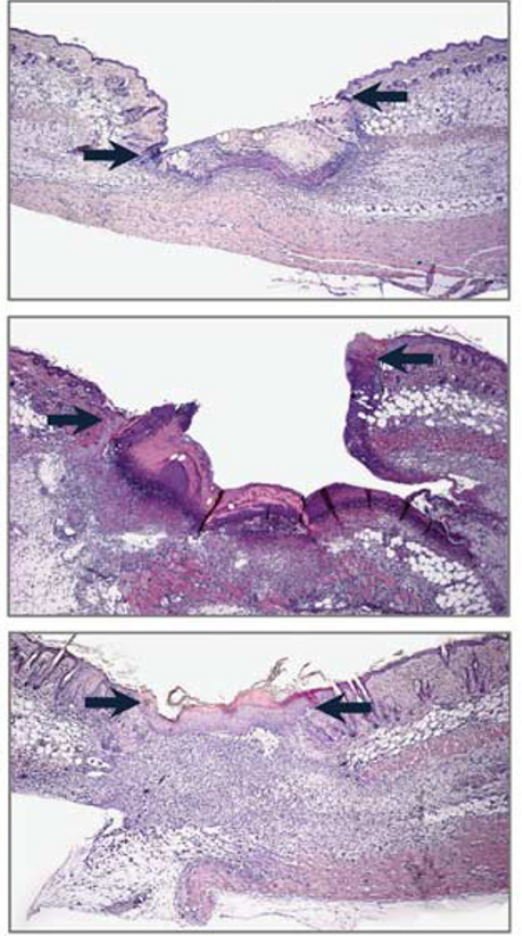

d
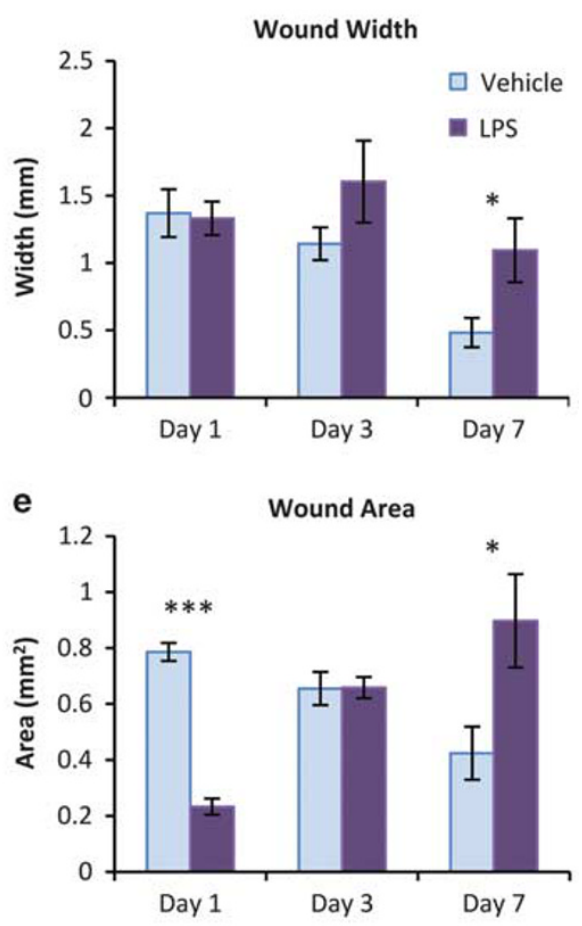

Figure 1 Application of LPS significantly delays cutaneous healing. (a) Representative photographs showing macroscopic excisional wound closure of C57 BL6 mice subcutaneously injected with PBS or $10 \mu \mathrm{g}$ LPS (scale bar $=5 \mathrm{~mm}$ ). (b) Planimetric analysis of wound photographs reveals significantly delayed healing in LPS-injected mice at 5-9 days postwounding. (c) Representative H\&E-stained sections of LPS- or PBS-injected incisional wounds, arrows indicate wound margins. (scale $=400 \mu \mathrm{m}$ ). Analysis of histological wound width (d) and area (e) reveals LPS significantly delays healing compared with PBS control wounds at 7 days and 1 and 7 days postwounding, respectively. Mean \pm s.e.m.; $n=5-6$ mice/group; ${ }^{*} P<0.05 ;{ }^{* *} P<0.01 ;{ }^{* * *} P<0.001$. 
a

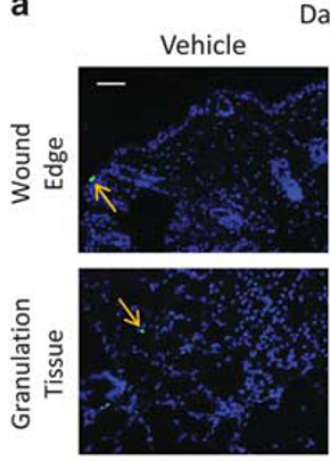

C

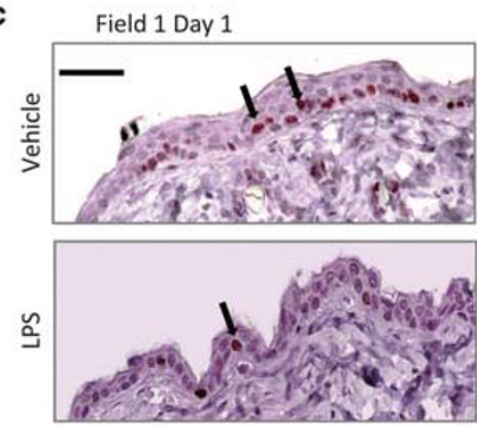

Day 1
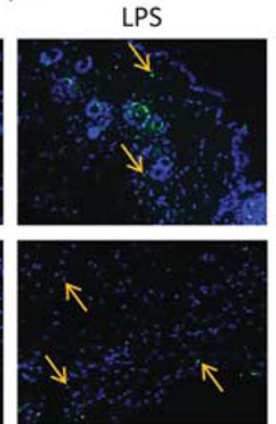

d

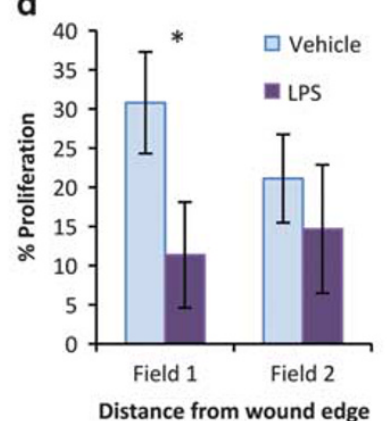

Day 3

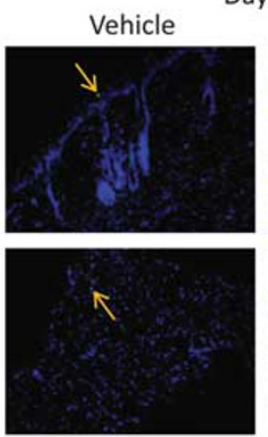

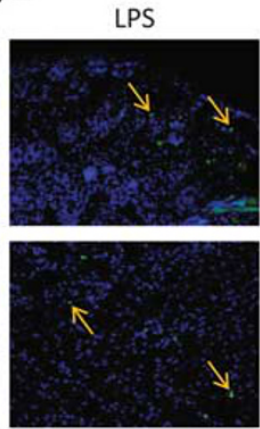

e

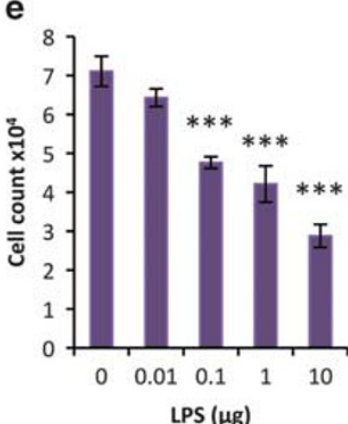

b

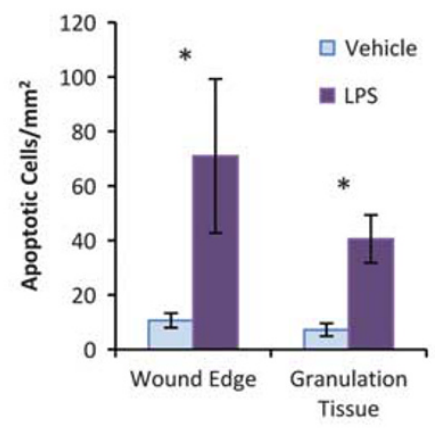

$\mathbf{f}$

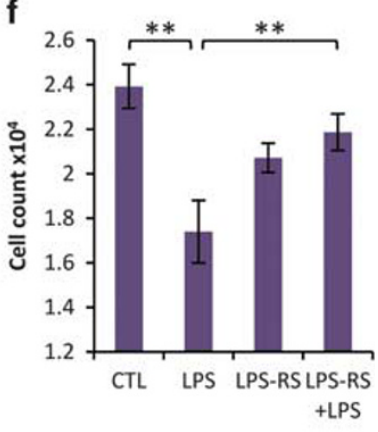

Figure 2 LPS induces early cell death and inhibits/delays wound edge proliferation. (a) Representative TUNEL staining of PBS- and LPS-injected wounds at 1 and 3 days postwounding. FITC (green)= cells undergoing apoptosis, arrows indicate representative positive stain. DAPI (blue) = nuclear stain. Scale $=50 \mu \mathrm{m}$. (b) Quantification shows LPS significantly increases apoptosis, both at the wound edge and within the granulation tissue, 1 day after wounding compared with PBS control wounds. (c) Representative immunohistochemistry (Ki67) of day 1 LPS vs PBS control wounds showing proliferation up to $500 \mu \mathrm{m}$ from the wound edge, named field 1. Arrows indicate representative positive stain. Scale $=50 \mu \mathrm{m}$. (d) Analysis of proliferating basal keratinocytes shows a significantly lower percentage of Ki67-positive basal cells directly at the wound edge (field 1) 1 day after wounding. Field $1=0-500 \mu \mathrm{m}$ from the wound edge, field $2=500-1000 \mu \mathrm{m}$ from the wound edge. A dose-dependent decrease in proliferation is also observed in NHEK cells when stimulated with LPS for $24 \mathrm{~h}$ in vitro (e). This effect is reversed with the addition of TLR4 antagonist, LPS-RS (f). Mean \pm s.e.m.; $n=5-6$ mice/group (a-d) and 7-8 replicates (e and f) ${ }^{*} P<0.05 ;{ }^{*} P<0.01 ;{ }^{* *} P<0.001$.

\section{LPS Leads to a Prolonged Inflammatory Response and Reduced Wound Collagen Deposition}

LPS and TLR signalling has been widely reported to activate the immune response and pro-inflammatory reactions. Moreover, excessive inflammation is a key driver of chronic wound aetiology. Intriguingly, early in healing (day 1 postwounding) LPS-treated wounds displayed a markedly dampened local recruitment of both neutrophils and macrophages (Figures $4 \mathrm{a}-\mathrm{c}$ ). By day 3, this was completely reversed with significantly heightened neutrophil $(P=0.005)$ and macrophage $(P=0.009)$ numbers in LPS wounds, with delayed macrophage resolution at day 7 . Moreover, at the transcriptional level, neutrophil (cx family) and leukocyte/ monocyte (cc family) chemokines displayed a corresponding profile of reduced early (day 1 ), but excessive late (day 3), expression in LPS-treated wounds (data not shown). The heightened influx of immune cells 3 days after wounding correlates with significantly increased pro-inflammatory cytokines, IL6 and TNF $\alpha$ within LPS-treated wounds after 3 days (Figures $5 f$ and $\mathrm{g}$ ). Immune-cell-mediated matrix metalloprotease (MMP) production (particularly MMP2 and MMP9) is increased in human chronic wounds. We observed a corresponding increase in transcription of MMP2, but not of MMP9, in LPS-treated wounds at 3 days after wounding (data not shown). In the early proliferative phase of healing, tightly regulated matrix deposition and remodelling is essential. We thus tested the hypothesis that MMP alterations would lead to altered wound matrix levels in LPS-treated wounds. Masson's Trichrome revealed a marked reduction in wound collagen (blue staining) in LPS wounds at both 3 and 7 days compared with vehicle (Figure 4d). Quantitative Picro-Sirius Red visualisation of day 7 LPS and vehicle wounds confirmed reduced collagen content. Moreover, significantly fewer thick (more structurally developed; red stained) collagen fibres were present in LPS day 7 wounds compared with vehicle. No significant difference was observed in the newly deposited (finer; green stained) collagen fibres (Figures $4 \mathrm{e}$ and $\mathrm{f}$ ).

\section{Oestrogen Treatment Partially Reverses LPS-Induced Delayed Healing}

Numerous studies have documented the beneficial effects of $17 \beta$-estradiol on wound healing (reviewed in Rieger et $a l^{52}$ ). To determine whether oestrogen could correct the 
a

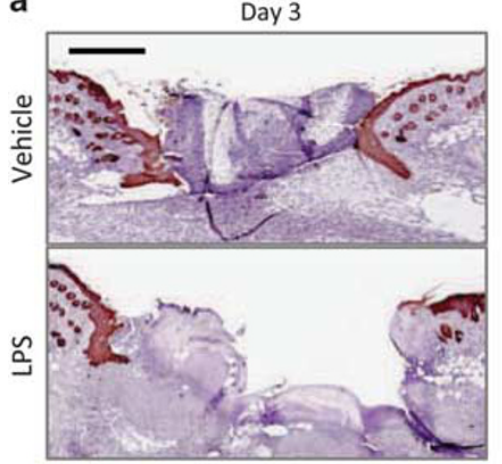

b

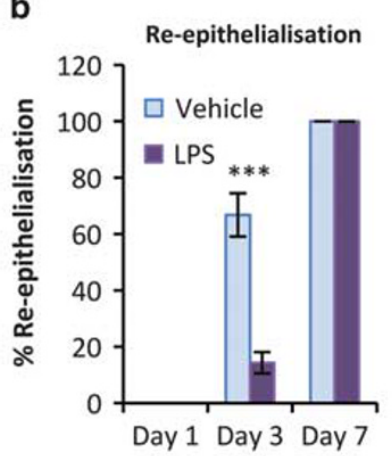

c

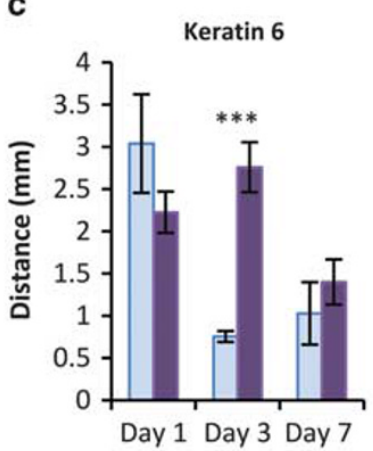

e

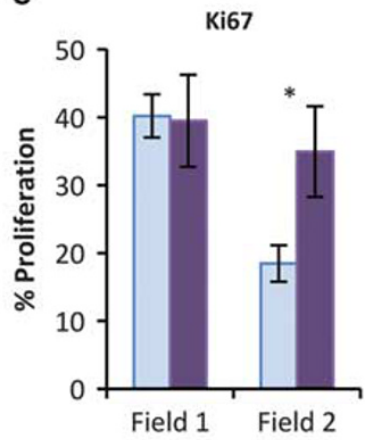

d
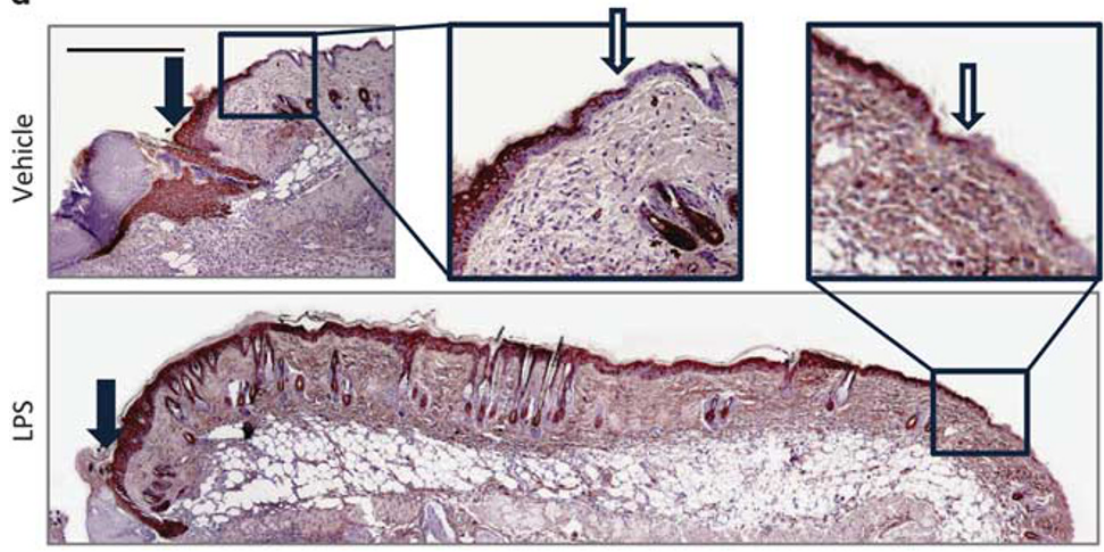

f

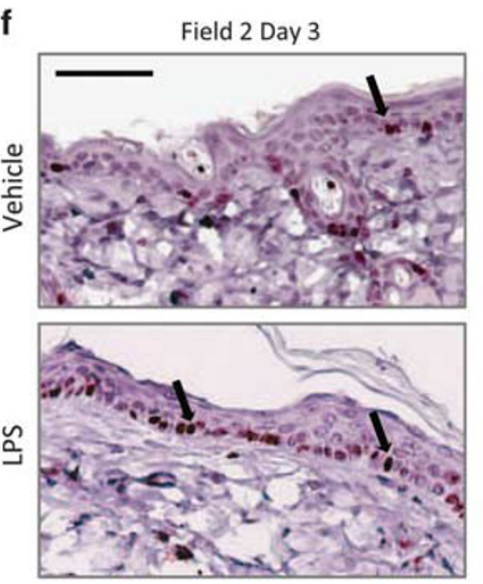

Figure 3 Delayed re-epithelialisation of LPS-treated wounds. (a) Representative immunohistochemistry (keratin 14) of PBS- and LPS-injected wounds at 3 days postwounding. Scale bar $=400 \mu \mathrm{m}$. (b) LPS significantly impairs re-epithelialisation at 3 days after wounding compared with PBS control wounds. (c) Analysis of keratin 6 expressing epidermal keratinocytes shows a significant increase in the distance contribution to migration/proliferation in LPS-treated wounds, 3 days after wounding, illustrated by immunohistochemical; keratin 6-stained images of PBS and LPS injected wounds at 3 days postwounding (d). Solid arrows indicate the wound edge; open arrows indicate the cessation of keratin 6 expression. Scale bar $=400 \mu \mathrm{m}$. (e) Quantification of proliferating basal keratinocytes shows a significantly higher percentage of Ki67-positive basal cells extending further from the wound edge, (field 2), at 3 days after wounding (field 1=0-500 $\mu \mathrm{m}$, field 2=500-1000 $\mu \mathrm{m}$ from the wound edge). (f) Representative immunohistochemical Ki67-stained images of LPS vs PBS control wounds in field 2. Arrows indicate representative positive stain. Bar $=50 \mu \mathrm{m}$. Mean \pm s.e.m.; $n=5-6$ mice/ group; ${ }^{* *} P<0.001 ;{ }^{*} P<0.05$.

LPS-induced delayed healing phenotype, we combined local LPS administration with systemic $17 \beta$-estradiol treatment. Oestrogen restored healing in the LPS model, although only partially. Specifically, 17 $\beta$-estradiol co-treatment improved wound closure while fully restoring the delay in re-epithelialisation $(P=0.003$; Figures $5 \mathrm{a}-\mathrm{c})$. In vitro assessment of oestrogen-treated human keratinocytes revealed this restoration is not due to a direct effect on proliferation (data not shown). In line with oestrogen's well-documented anti-inflammatory activity, wound macrophage infiltration was significantly reduced in co-treated wounds $v s$ LPS alone $(P=0.021$; Figures $5 \mathrm{~d}$ and $\mathrm{e})$, whereas intriguingly no difference was observed in wound neutrophil numbers (data not shown). Moreover, LPS-induced IL6 and TNF $\alpha$ levels were fully restored to vehicle levels by oestrogen treatment (Figures $5 \mathrm{f}$ and g). Finally, as $17 \beta$-estradiol has previously been shown to directly induce fibroblast matrix production, we assessed the level of wound collagen deposition. Here we report that oestrogen co-treatment partially restores wound granulation tissue collagen $v s$ LPS-treated wounds alone (Figures $5 \mathrm{~h}$ and i). These data collectively demonstrate the value of this LPS-induced delayed healing model, revealing potential beneficial effects of oestrogen treatment on wounds with high bacterially induced TLR4 activation.

\section{DISCUSSION}

Wound infection is increasingly cited as a major causative factor in the persistence of chronic wounds. ${ }^{53}$ The bacterial diversity of pathogenic biofilm communities in chronic wounds is vast and thought to vary dependent on wound type. Although chronic wounds contain both Gram-positive and Gram-negative species, the latter predominate, particularly in venous leg ulcers. ${ }^{54}$ At the molecular level, an organism senses and responds to wound bacteria (ie, infection) via PRRs, initiating a highly orchestrated host immune response. LPS, the major component of all 
a

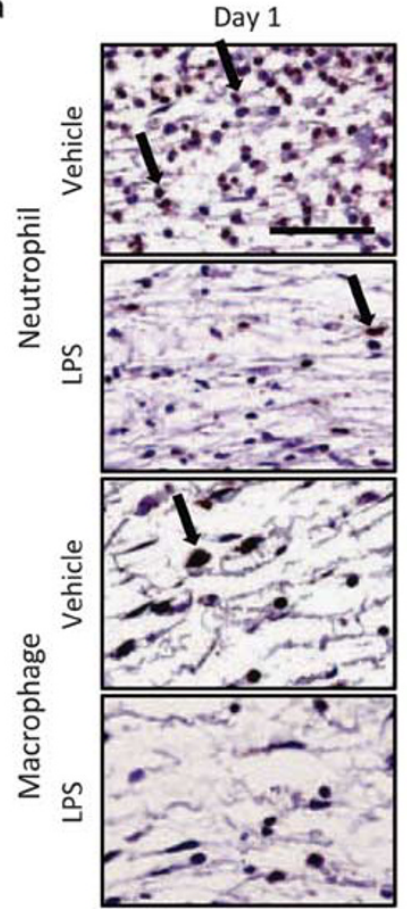

d
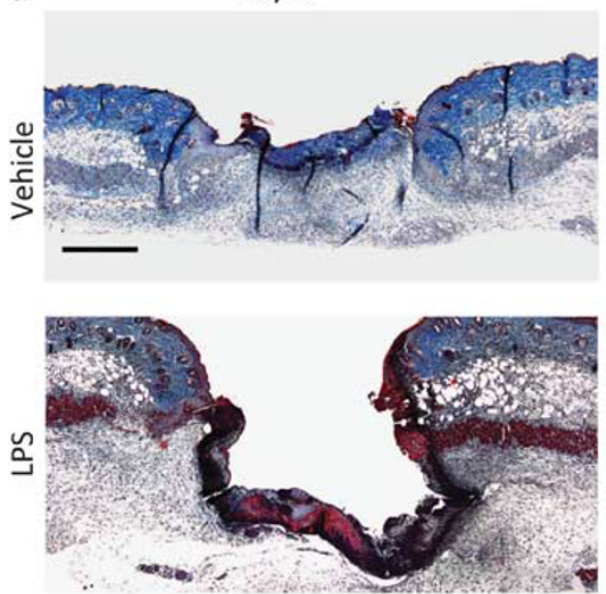

e

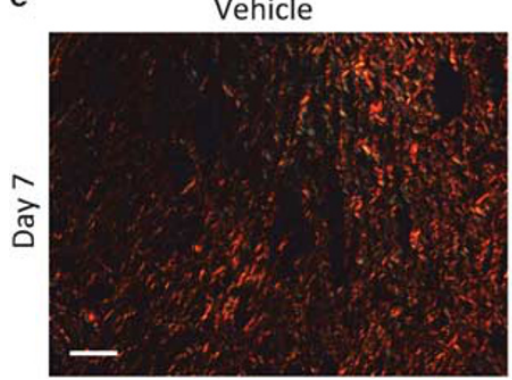

Day 3

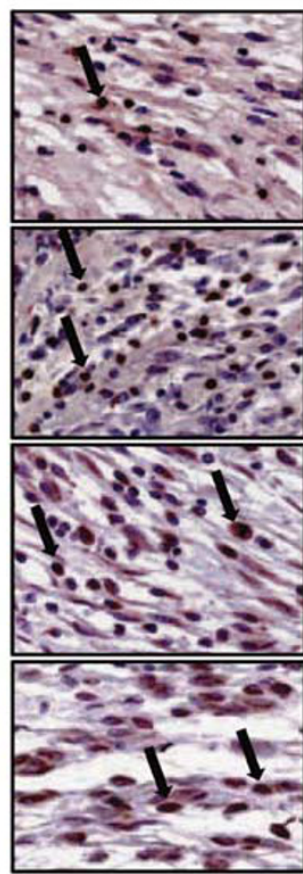

Day 7

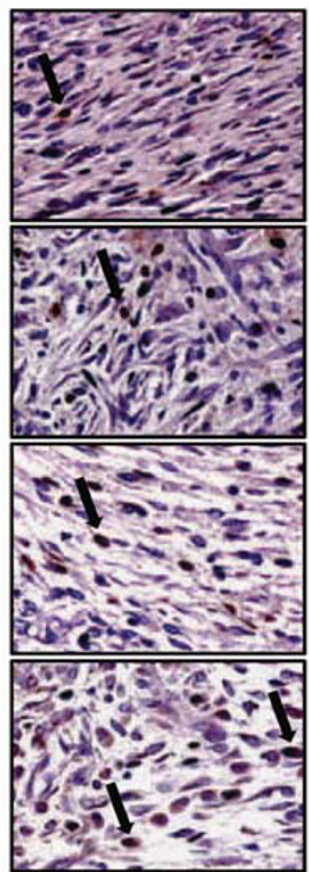

b
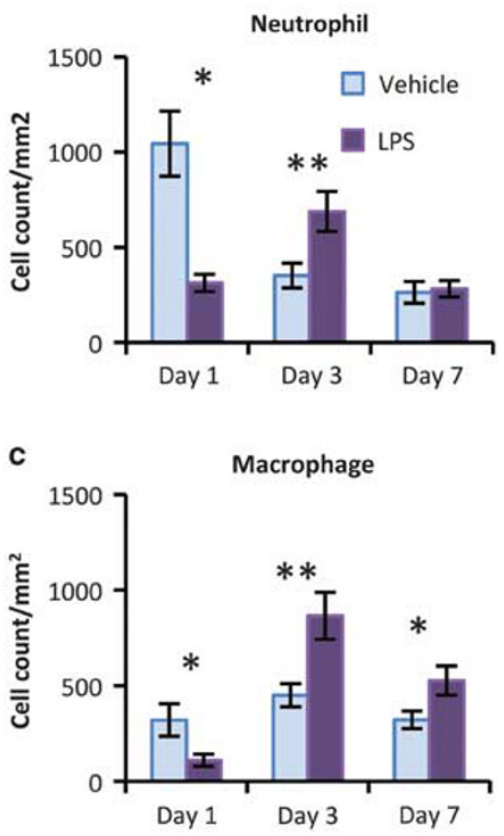

Day 7
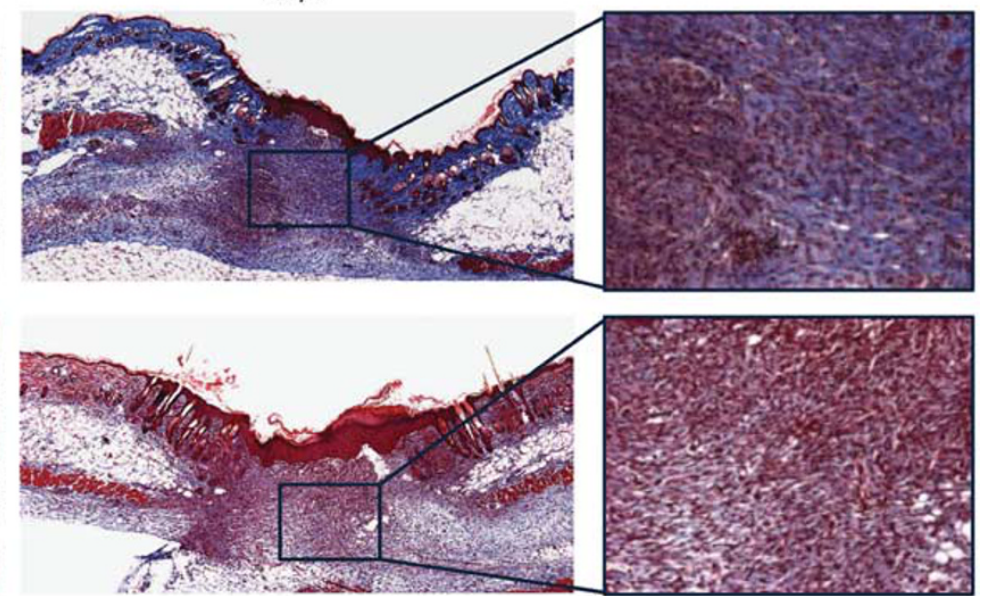

LPS

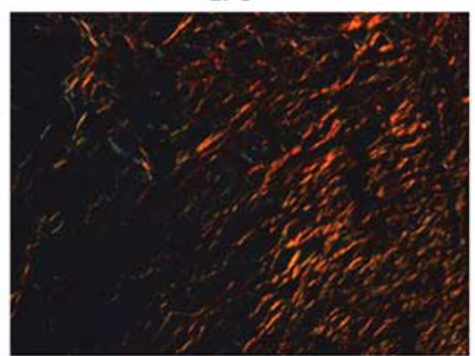

f

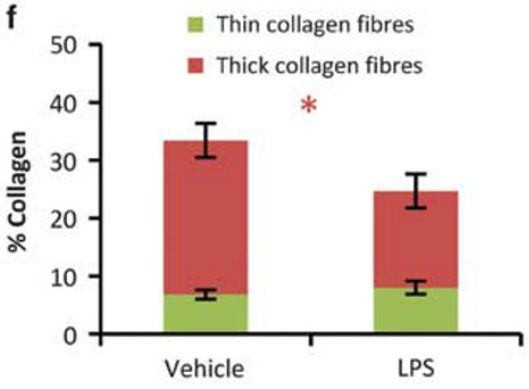

Figure 4 LPS causes a delayed, prolonged inflammatory response, resulting in reduced collagen deposition. (a) Representative immunohistochemistry of immune cell infiltration within LPS-injected incisional wounds over a time course of 1, 3 and 7 days after wounding. Arrows indicate representative positive stain. Scale $=50 \mu \mathrm{m}$. (b and $\mathbf{c}$ ) Quantification of neutrophils and macrophages reveals a significant reduction in LPS-treated wounds at 1 day postwounding, reversed by day 3 where we see heightened inflammatory cell counts and macrophage numbers remaining high at day 7. (d) Representative Masson's Trichrome-stained wound sections from mice subjected to LPS or PBS, collected 3 or 7 days after wounding. Scale $=400 \mu \mathrm{m}$. (e) Picro-Sirius Red staining of day 7 LPS and control wounds. Scale $=100 \mu \mathrm{m}$. (f) Quantification reveals an overall reduction of collagen content, specifically significantly less thicker, red stained, collagen fibres present in LPS 7 day wounds compared with control. Mean + s.e.m.; $n=5-6$ mice/group; ${ }^{* *} P<0.01 ;{ }^{*} P<0.05$. 
Gram-negative bacterial cell walls, activates PRR TLR4. Studies suggest that in humans TLR4 is central to this process, with continuous TLR4 stimulation observed in non-healing chronic wounds, ${ }^{17}$ likely the result of increased bacterial load. Moreover, mechanistic studies using TLR4 null mice have shown TLR4 to be important in the early stages a

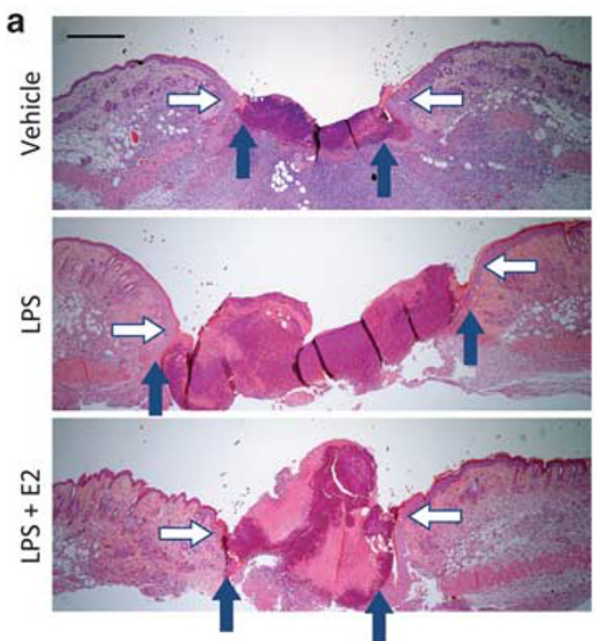

d
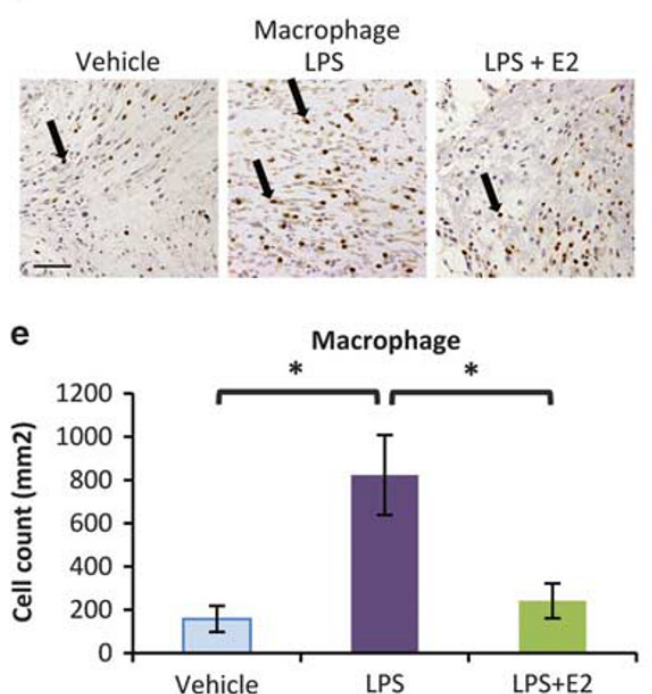

h

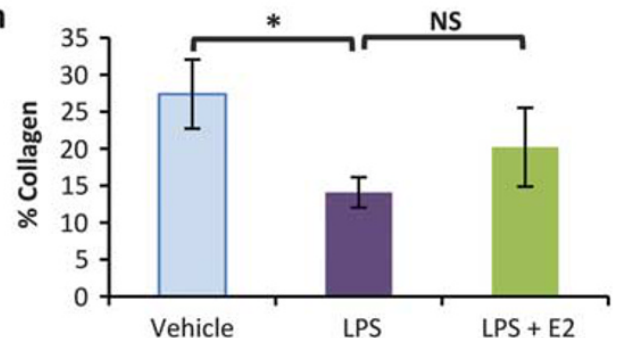

b
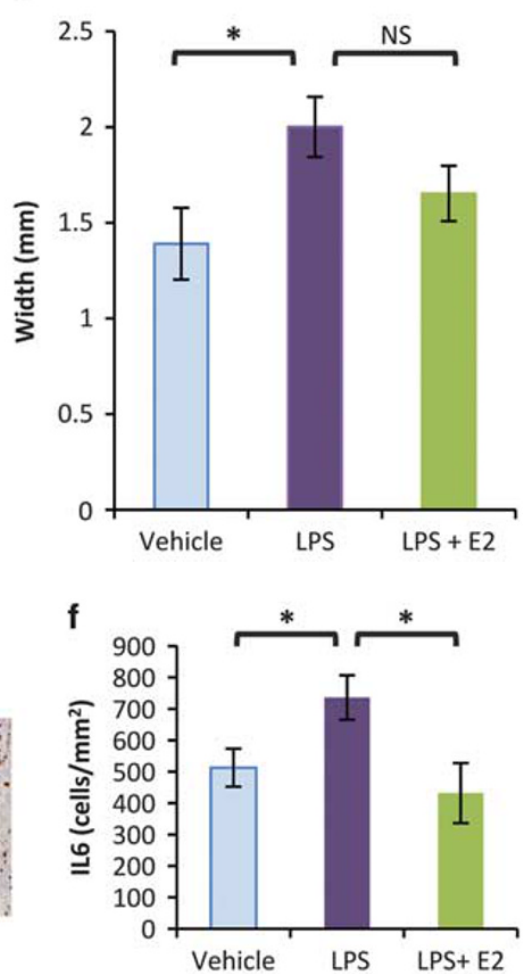

i
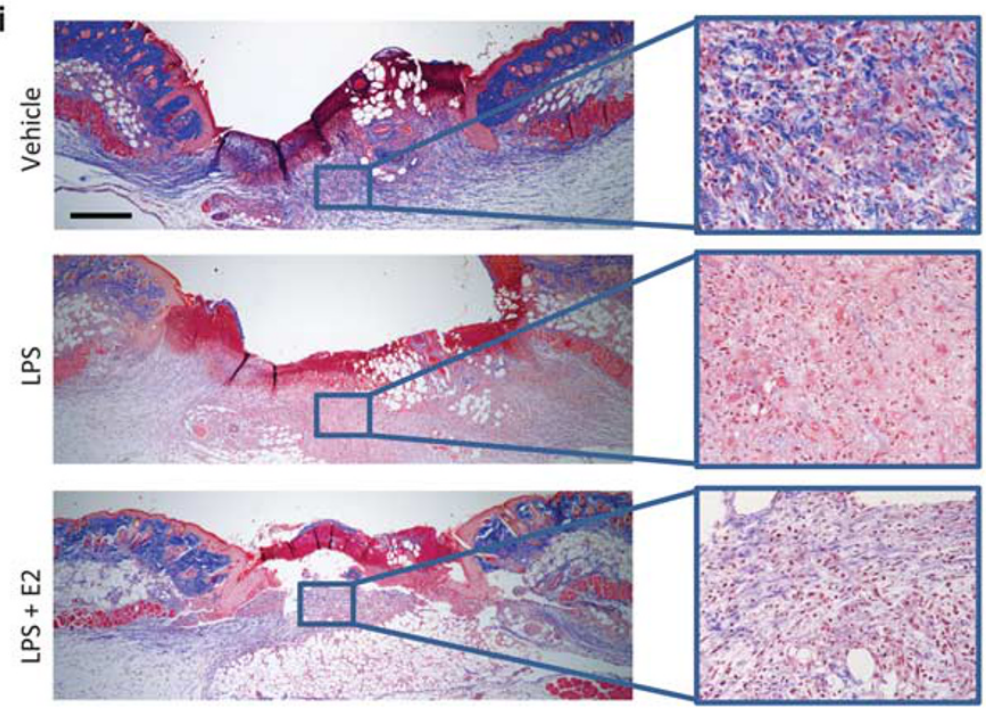

Figure 5 Oestrogen partially restores the LPS-induced delay in cutaneous healing. (a) Representative H\&E-stained sections of vehicle-, LPS- and LPS +oestrogen-injected incisional wounds collected 3 days after wounding, open arrows indicate wound margins, solid arrows indicate leading neo-epidermal edge $($ scale $=400 \mu \mathrm{m})$. Analysis of histological wound width $(\mathbf{b})$ and the percentage of re-epithelialisation (c) reveals oestrogen fully restores the delayed re-epithelialisation caused by LPS. (d) Representative immunohistochemistry of macrophage infiltration within vehicle-, LPS- and LPS+oestrogen-injected incisional wounds 3 days after wounding. Arrows indicate representative positive stain (scale $=50 \mu \mathrm{m}$ ). Quantification reveals a significant reduction of macrophages in LPS+E2-treated wounds compared with LPS (e). Quantification of immunohistochemical staining reveals increased numbers of IL6 (f) and TNF $a(\mathbf{g})$ expressing cells in wound granulation tissue induced by LPS, fully restored to vehicle levels by the presence of oestrogen. (i) Representative Masson's Trichrome staining of vehicle-, LPS- and LPS+oestrogen-treated incisions. Quantification shows that the LPS effect of reduced collagen deposition is partially restored by the presence of oestrogen (h). Scale $=400 \mu$ m. Mean \pm s.e.m.; $n=8$ mice/group; ${ }^{*} P<0.05 ;{ }^{* *} P<0.01 ;{ }^{* * *} P<0.001$. 
of wound repair. ${ }^{41}$ Here we report that direct wound administration of LPS, derived from $K$. pneumoniae, an emerging wound pathogen, leads to a pronounced delay in healing. Specifically, LPS-treated wounds displayed increased local cell death, reduced proliferation, delayed but ultimately heightened immune cell recruitment and pro-inflammatory signals and reduced matrix deposition. We note that our observed phenotype is in agreement with other local and systemic LPS administration models. For example, local and systemic LPS as a model of infection in burn injury induced a heightened inflammatory response and epidermal thickening, ${ }^{35,55,56}$ whereas decreased collagen deposition was observed with systemic administration of LPS in cutaneous rat wounds. ${ }^{57}$ Crucially, we demonstrate the utility of LPS administration as a delayed healing model by showing that co-administration of the pleiotropic healing-promoting factor $17 \beta$-estradiol is able to reverse some, but not all, aspects of the LPS healing phenotype.

Collectively, our data suggest that local LPS administration has a dramatic immediate effect on the early stages of wound repair, a phenotype that is qualitatively very different from that of other delayed/chronic wound models (eg, db/db mice). In essence, LPS appears to block initiation of the healing response, with LPS-administered wounds failing to show macroscopic signs of local cellular activation (eg, wound edge thickening) in the first $24 \mathrm{~h}$ postwounding. This is confirmed histologically, with wounds subjected to LPS displaying reduced wound edge proliferation, an important aspect of injury-induced wound activation. Importantly, our in vitro data show decreased proliferation to be a keratinocyte intrinsic response to TLR4 stimulation by LPS (Figure 2e). We note that airway epithelial cells in vitro display decreased proliferation when treated with LPS derived from $P$. aeruginosa, although in these cells low doses induce proliferation. ${ }^{58}$ LPS derived from $P$. aeruginosa and Escherichia coli have been previously shown to directly inhibit human keratinocyte migration in vitro ${ }^{59}$; however, we found LPS derived from $K$. pneumoniae induced no effect on human keratinocyte scratch wound closure. Histologically, LPS-administered wound tissue appears necrotic with decreased cellularity, mirroring a phenotype previously reported by Ishikawa et $a^{38}$ following Salmonella and E. coli-derived LPS application in unwounded skin. We show that LPS administration directly induces local apoptosis in vivo (Figure 2), in line with reports of bacterial-induced keratinocyte apoptosis in vitro. ${ }^{60}$ High levels of local cell death may lead to the release of DAMPs, which will likely in turn activate other PRRs to perpetuate the phenotype. Intriguingly, we note that prior studies have also reported beneficial effects of non-Klebsiella-derived LPS on wound healing. ${ }^{58,61}$ It remains unclear how LPS confers beneficial effects in the context of these studies.

Keratin 6 (K6), a marker of activated wound edge keratinocytes, has been shown to have important structural and signalling roles, the latter regulating cellular proliferation and migration. ${ }^{62}$ A common characteristic of delayed healing wounds is epidermal hyper-proliferation and migration inhibition. ${ }^{63}$ In the LPS model, we report markedly increased proliferation at the wound periphery coinciding with a significant delay in re-epithelialisation, accompanied by extended peripheral cell activation of the epidermis. This presumably reflects a mechanism to compensate for the lack of initial wound activation. Alternatively, a recently reported mechanistic link between K6 and PRRs suggests a more direct association. Keratins assemble as pairs, with keratin 16, the type 1 keratin partner of K6, required for the regulation of DAMP expression. ${ }^{64}$ Specifically, K16 deficiency leads to cytokine and DAMP overexpression. It is therefore possible that extended expression of these 'wound-type' keratins in LPS-treated wounds may have evolved to suppress a heightened inflammatory response to skin bacteria upon injury.

Oestrogen deficiency is a known risk factor for human chronic wounds. ${ }^{44}$ Indeed, the beneficial effects of oestrogen on healing have been demonstrated across a range of pathological skin wound models, including aged and ovariectomised mice, ${ }^{46,47}$ type ${ }^{65}$ and type 2 diabetes ${ }^{66}$ and the skin flap ischemia model, ${ }^{67}$ where oestrogen has been shown to dampen the local immune response, promote keratinocyte migration and proliferation and induce fibroblast-mediated matrix deposition. ${ }^{46}$ In addition, oestrogenic effects on thermal injury have been well studied, for example, in the brain, where reduced inflammation and apoptosis are linked to lower levels of DAMPs. ${ }^{68,69}$ Although oestrogen has been shown to promote acute wound healing in humans, ${ }^{45}$ no data exist for its effect on infected cutaneous wounds. Here we show that oestrogen exerts beneficial effects on both epidermal and dermal aspects of cutaneous healing in a local LPS bacterial infection model.

We note that our data are in line with previous studies demonstrating the beneficial effects of oestrogen treatment in both systemic and local LPS inflammation models. These include systemic LPS-induced brain inflammation in ovariectomised mice, ${ }^{70}$ LPS-induced lung injury in rats ${ }^{71,72}$ and a well-characterised trauma-haemorrhage model which demonstrated that $17 \beta$-estradiol restores the epidermal keratinocyte inflammatory response by preventing TLR4-mediated MAPK activation. ${ }^{73}$ In our study, we show that oestrogen dampens the LPS-induced inflammatory signals via IL6 and TNF $\alpha$ with reduced wound macrophage infiltration, mirroring results previously demonstrated in vitro in LPS-stimulated macrophages treated with $17 \beta$-estradiol. ${ }^{74}$ In addition to oestrogen's well-documented anti-inflammatory properties, previous mechanistic studies suggest a direct influence on wound re-epithelialisation. Previous studies demonstrate increased migration but not proliferation of oestrogen-treated murine keratinocytes in vitro, in line with our in vitro data. ${ }^{74,75}$

In summary, our data demonstrate that locally applied K. pneumoniae-derived LPS delays multiple aspects of 
cutaneous healing, inducing excessive cell death, stalling keratinocyte activation, heightening the local inflammatory response and reducing collagen deposition. We suggest that LPS administration could be adopted as a simple model of bacterial wound infection. We note that, although this model does not consider major LPS differences between strains or other bacterial components, by-passing the need for live bacteria to generate a highly reproducible and defined delayed healing model is a major advantage. A future, extremely complex, goal will be to produce a faithful model of chronic wound infection, encompassing the plethora of microorganisms present clinically in wound biofilms. ${ }^{76}$ In this study, we demonstrate the utility of our LPS model to explore the biological effects of oestrogen on 'infected' wounds. Indeed, these data provide the first evidence that oestrogen's beneficial influence on healing should apply to both infected and non-infected wounds, which has major clinical implications.

\section{ACKNOWLEDGMENTS}

We thank Kelly Wemyss, Charlotte Marsh and Peter Walker for technical assistance. This work was funded by the MRC. The Bioimaging Facility microscopes used in this study were purchased with grants from BBSRC, Wellcome and the University of Manchester Strategic Fund. This research was supported by grants from the Medical Research Council to MJH and SC and InnovateUK, Epistem and AgeUK to MJH.

\section{DISCLOSURE/CONFLICT OF INTEREST}

The authors declare no conflict of interest.

1. Human Microbiome Project C. Structure, function and diversity of the healthy human microbiome. Nature 2012;486:207-214.

2. Harder J, Bartels J, Christophers E et al. A peptide antibiotic from human skin. Nature 1997;387:861.

3. Belkaid Y, Segre JA. Dialogue between skin microbiota and immunity. Science 2014;346:954-959.

4. Gontcharova V, Youn E, Sun $Y$ et al. A comparison of bacterial composition in diabetic ulcers and contralateral intact skin. Open Microbiol J 2010;4:8-19.

5. Martin JM, Zenilman JM, Lazarus GS. Molecular microbiology: new dimensions for cutaneous biology and wound healing. J Invest Dermatol 2010;130:38-48.

6. Hart J. Inflammation. 2: Its role in the healing of chronic wounds. J Wound Care 2002:11:245-249.

7. Custovic A, Smajlovic J, Hadzic S et al. Epidemiological surveillance of bacterial nosocomial infections in the surgical intensive care unit. Mater Sociomed 2014;26:7-11.

8. Calhoun JH, Murray CK, Manring MM. Multidrug-resistant organisms in military wounds from Iraq and Afghanistan. Clin Orthop Relat Res 2008;466:1356-1362.

9. Petersen K, Riddle MS, Danko JR et al. Trauma-related infections in battlefield casualties from Iraq. Ann Surg 2007;245:803-811.

10. Yun HC, Murray CK, Roop SA et al. Bacteria recovered from patients admitted to a deployed U.S. military hospital in Baghdad, Iraq. Mil Med 2006;171:821-825.

11. Weintrob AC, Roediger MP, Barber $M$ et al. Natural history of colonization with gram-negative multidrug-resistant organisms among hospitalized patients. Infect Control Hosp Epidemiol 2010;31:330-337.

12. Mayhall CG. The epidemiology of burn wound infections: then and now. Clin Infect Dis 2003;37:543-550.

13. Oates A, Bowling FL, Boulton AJ et al. Molecular and culture-based assessment of the microbial diversity of diabetic chronic foot wounds and contralateral skin sites. J Clin Microbiol 2012;50:2263-2271.
14. Wysocki AB, Bhalla-Regev SK, Tierno Jr PM et al. Proteolytic activity by multiple bacterial species isolated from chronic venous leg ulcers degrades matrix substrates. Biol Res Nurs 2013;15:407-415.

15. Yoga $R$, Khairul A, Sunita $K$ et al. Bacteriology of diabetic foot lesions. Med J Malaysia 2006;61(Suppl A):14-16.

16. Varaiya AY, Dogra JD, Kulkarni MH et al. Extended-spectrum betalactamase-producing Escherichia coli and Klebsiella pneumoniae in diabetic foot infections. Indian J Pathol Microbiol 2008;51:370-372.

17. Pukstad BS, Ryan L, Flo TH et al. Non-healing is associated with persistent stimulation of the innate immune response in chronic venous leg ulcers. J Dermatol Sci 2010;59:115-122.

18. Schierle CF, De la Garza M, Mustoe TA et al. Staphylococcal biofilms impair wound healing by delaying reepithelialization in a murine cutaneous wound model. Wound Repair Regen 2009;17:354-359.

19. Scales BS, Huffnagle GB. The microbiome in wound repair and tissue fibrosis. J Pathol 2013;229:323-331.

20. Zhao G, Usui ML, Lippman SI et al. Biofilms and inflammation in chronic wounds. Adv Wound Care (New Rochelle) 2013;2:389-399.

21. Bosanquet DC, Harding KG. Wound duration and healing rates: cause or effect? Wound Repair Regen 2014;22:143-150.

22. Gardner SE, Frantz RA. Wound bioburden and infection-related complications in diabetic foot ulcers. Biol Res Nurs 2008;10:44-53.

23. Mills Sr JL, Conte MS, Armstrong DG et al. The Society for Vascular Surgery Lower Extremity Threatened Limb Classification System: risk stratification based on wound, ischemia, and foot infection (WIfl). J Vasc Surg 2014;59:220-234.

24. Kumar S, Ingle H, Prasad DV et al. Recognition of bacterial infection by innate immune sensors. Crit Rev Microbiol 2013;39:229-246.

25. Kawai K, Shimura H, Minagawa $M$ et al. Expression of functional Tolllike receptor 2 on human epidermal keratinocytes. J Dermatol Sci 2002;30:185-194.

26. Wang J, Hori K, Ding J et al. Toll-like receptors expressed by dermal fibroblasts contribute to hypertrophic scarring. J Cell Physiol 2011;226: 1265-1273.

27. West AP, Brodsky IE, Rahner $\mathrm{C}$ et al. TLR signalling augments macrophage bactericidal activity through mitochondrial ROS. Nature 2011;472:476-480.

28. Dasu MR, Martin SJ. Toll-like receptor expression and signaling in human diabetic wounds. World J Diabetes 2014;5:219-223.

29. Lin $Q$, Fang $D$, Fang J et al. Impaired wound healing with defective expression of chemokines and recruitment of myeloid cells in TLR3-deficient mice. J Immunol 2011;186:3710-3717.

30. Sato T, Yamamoto M, Shimosato $T$ et al. Accelerated wound healing mediated by activation of Toll-like receptor 9 . Wound Repair Regen 2010;18:586-593.

31. Campbell L, Williams $\mathrm{H}$, Crompton RA et al. Nod2 deficiency impairs inflammatory and epithelial aspects of the cutaneous wound-healing response. J Pathol 2013;229:121-131.

32. Macedo L, Pinhal-Enfield G, Alshits $V$ et al. Wound healing is impaired in MyD88-deficient mice: a role for MyD88 in the regulation of wound healing by adenosine A2A receptors. Am J Pathol 2007;171: 1774-1788.

33. Dasu MR, Thangappan RK, Bourgette $A$ et al. TLR2 expression and signaling-dependent inflammation impair wound healing in diabetic mice. Lab Invest 2010;90:1628-1636.

34. Lin $Q$, Wang $L$, Lin $Y$ et al. Toll-like receptor 3 ligand polyinosinic: polycytidylic acid promotes wound healing in human and murine skin. J Invest Dermatol 2012;132:2085-2092.

35. Konturek PC, Brzozowski T, Konturek SJ et al. Influence of bacterial lipopolysaccharide on healing of chronic experimental ulcer in rat. Scand J Gastroenterol 2001;36:1239-1247.

36. Brothers KM, Stella NA, Hunt KM et al. Putting on the brakes: Bacterial impediment of wound healing. Sci Rep 2015;5:14003.

37. Stifano G, Affandi AJ, Mathes AL et al. Chronic Toll-like receptor 4 stimulation in skin induces inflammation, macrophage activation, transforming growth factor beta signature gene expression, and fibrosis. Arthritis Res Ther 2014;16:R136.

38. Ishikawa $Y$, Kirikae $T$, Hirata $M$ et al. Local skin response in mice induced by a single intradermal injection of bacterial lipopolysaccharide and lipid A. Infect Immun 1991;59:1954-1960.

39. Wichterman KA, Baue $A E$, Chaudry IH. Sepsis and septic shock-a review of laboratory models and a proposal. J Surg Res 1980;29: 189-201. 
40. Suga $H$, Sugaya $M$, Fujita $H$ et al. TLR4, rather than TLR2, regulates wound healing through TGF-beta and CCL5 expression. J Dermatol Sci 2014;73:117-124.

41. Chen L, Guo S, Ranzer MJ et al. Toll-like receptor 4 plays an essential role in early skin wound healing. J Invest Dermatol 2013;133:258-267.

42. Singh K, Singh VK, Agrawal NK et al. Association of Toll-like receptor 4 polymorphisms with diabetic foot ulcers and application of artificial neural network in DFU risk assessment in type 2 diabetes patients. Biomed Res Int 2013;2013.

43. Ansell DM, Holden KA, Hardman MJ. Animal models of wound repair: are they cutting it? Exp Dermatol 2012;21:581-585.

44. Margolis DJ, Knauss J, Bilker W. Hormone replacement therapy and prevention of pressure ulcers and venous leg ulcers. Lancet 2002;359: 675-677.

45. Ashcroft GS, Greenwell-Wild T, Horan MA et al. Topical estrogen accelerates cutaneous wound healing in aged humans associated with an altered inflammatory response. Am J Pathol 1999;155:1137-1146.

46. Emmerson E, Campbell L, Ashcroft GS et al. Unique and synergistic roles for 17beta-estradiol and macrophage migration inhibitory factor during cutaneous wound closure are cell type specific. Endocrinology 2009:150:2749-2757.

47. Mukai $K$, Nakajima $Y$, Urai $T$ et al. 17beta-Estradiol administration promotes delayed cutaneous wound healing in 40-week ovariectomised female mice. Int Wound J 2014, Aug 6. doi:10.1111/iwj.12336. [e-pub ahead of print], PMID: 25132513.

48. Ansell DM, Campbell L, Thomason HA et al. A statistical analysis of murine incisional and excisional acute wound models. Wound Repair Regen 2014;22:281-287.

49. Ashida H, Mimuro H, Ogawa $\mathrm{M}$ et al. Cell death and infection: a doubleedged sword for host and pathogen survival. J Cell Biol 2011;195: 931-942.

50. Ferluga J, Allison AC. Role of mononuclear infiltrating cells in pathogenesis of hepatitis. Lancet 1978;2:610-611.

51. Peavy DL, Baughn RE, Musher DM. Strain-dependent cytotoxic effects of endotoxin for mouse peritoneal macrophages. Infect Immun 1978;21:310-319.

52. Rieger $\mathrm{S}$, Zhao $\mathrm{H}$, Martin $\mathrm{P}$ et al. The role of nuclear hormone receptors in cutaneous wound repair. Cell Biochem Funct 2015;33:1-13.

53. Grice EA, Segre JA. Interaction of microbiome and the innate immune response in chronic wounds. Adv Exp Med Biol 2012;946:55-68.

54. Dowd SE, Sun Y, Secor PR et al. Survey of bacterial diversity in chronic wounds using Pyrosequencing, DGGE, and full ribosome shotgun sequencing. BMC Microbiol 2008;8:43.

55. Park BK, Kim D, Cho S et al. Effects of lipopolysaccharide and CpG-DNA on burn-induced skin injury. BMB Rep 2011;44:273-278.

56. Ami K, Kinoshita M, Yamauchi A et al. IFN-gamma production from liver mononuclear cells of mice in burn injury as well as in postburn bacterial infection models and the therapeutic effect of IL-18. $\mathrm{J}$ Immunol 2002;169:4437-4442.

57. Kawaguchi $\mathrm{H}$, Hizuta $\mathrm{A}$, Tanaka $\mathrm{N}$ et al. Role of endotoxin in wound healing impairment. Res Commun Mol Pathol Pharmacol 1995;89: 317-327.

58. Koff JL, Shao MX, Kim S et al. Pseudomonas lipopolysaccharide accelerates wound repair via activation of a novel epithelial cell signaling cascade. J Immunol 2006;177:8693-8700.
59. Loryman C, Mansbridge J. Inhibition of keratinocyte migration by lipopolysaccharide. Wound Repair Regen 2008;16:45-51.

60. Kirker KR, Secor PR, James GA et al. Loss of viability and induction of apoptosis in human keratinocytes exposed to Staphylococcus aureus biofilms in vitro. Wound Repair Regen 2009;17:690-699.

61. Kostarnoy AV, Gancheva PG, Logunov DY et al. Topical bacterial lipopolysaccharide application affects inflammatory response and promotes wound healing. J Interferon Cytokine Res 2013;33:514-522.

62. Morasso Ml, Tomic-Canic M. Epidermal stem cells: the cradle of epidermal determination, differentiation and wound healing. Biol Cell 2005;97:173-183.

63. Pastar I, Stojadinovic O, Yin NC et al. Epithelialization in wound healing: a comprehensive review. Adv Wound Care (New Rochelle) 2014;3: 445-464.

64. Lessard JC, Piña-Paz S, Rotty JD et al. Keratin 16 regulates innate immunity in response to epidermal barrier breach. Proc Natl Acad Sci USA 2013;110:19537-19542.

65. Tie $L, A n Y$, Han J et al. Genistein accelerates refractory wound healing by suppressing superoxide and FoxO1/iNOS pathway in type 1 diabetes. J Nutr Biochem 2013;24:88-96.

66. Pincus DJ, Kassira N, Gombosh $\mathrm{M}$ et al. 17ß-Estradiol modifies diabetic wound healing by decreasing matrix metalloproteinase activity. Wounds 2010;22:171-178.

67. Toutain CE, Brouchet L, Raymond-Letron I et al. Prevention of skin flap necrosis by estradiol involves reperfusion of a protected vascular network. Circ Res 2009;104:245-254.

68. Gatson JW, Maass DL, Simpkins JW et al. Estrogen treatment following severe burn injury reduces brain inflammation and apoptotic signaling. J Neuroinflammation 2009;6:30.

69. Yao X, Wigginton JG, Maass DL et al. Estrogen-provided cardiac protection following burn trauma is mediated through a reduction in mitochondria-derived DAMPs. Am J Physiol Heart Circ Physiol 2014;306:H882-H894.

70. Brown CM, Mulcahey TA, Filipek NC et al. Production of proinflammatory cytokines and chemokines during neuroinflammation: novel roles for estrogen receptors alpha and beta. Endocrinology 2010;151:4916-4925.

71. Zhou GJ, Zhang H, Zhi SD et al. Protective effect of raloxifene on lipopolysaccharide and acid- induced acute lung injury in rats. Acta Pharmacol Sin 2007;28:1585-1590.

72. $\mathrm{Li} \mathrm{X}, \mathrm{Xu} \mathrm{T}$, Lian $\mathrm{Q}$ et al. Protective effect of genistein on lipopolysaccharide-induced acute lung injury in rats. J Huazhong Univ Sci Technol Med Sci 2005;25:454-457.

73. Moeinpour F, Choudhry MA, Kawasaki $T$ et al. 17 Beta-estradiol normalizes Toll receptor 4, mitogen activated protein kinases and inflammatory response in epidermal keratinocytes following traumahemorrhage. Mol Immunol 2007;44:3317-3323.

74. Emmerson E, Campbell L, Ashcroft GS et al. The phytoestrogen genistein promotes wound healing by multiple independent mechanisms. Mol Cell Endocrinol 2010;321:184-193.

75. Campbell L, Emmerson E, Davies F et al. Estrogen promotes cutaneous wound healing via estrogen receptor beta independent of its antiinflammatory activities. J Exp Med 2010;207:1825-1833.

76. Seth AK, Geringer MR, Hong SJ et al. In vivo modeling of biofilminfected wounds: a review. J Surg Res 2012;178:330-338. 